\title{
Session 9: Anti-infective, anti-bacterial/viral antibodies: A new emerging paradigm in the field of anti-infectives - II
}

Friday 12 May, 2006. Moderator: Alex von Gabain

[14.30-14.50]

Human monoclonals in the fight against emerging infections: SARS, Avian Flu and West Nile virus Jan ter Meulen

CRUCELL Holland B.V., Leiden, The Netherlands

Abstract not received.

[14.50-15.10]

Anti-toxin anthrax antibodies

Israel Lowy

Medarex, Milpitas, CA, USA

Abstract not received.

\section{[15.10-15.30]}

Development of a human monoclonal antibody cocktail for use in rabies post-exposure

prophylaxis

Alexander B.H. Bakker

Crucell Holland B.V., Leiden, The Netherlands

Human mortality from canine rabies is estimated by World Health Organization (WHO) to be 55.000 deaths per year. Limited supply hampers the accessibility of appropriate life saving treatment, particularly in endemic areas. Anti-rabies immunoglobulin is currently used in combination with rabies vaccine to protect humans from lethal rabies. For cost and safety reasons, replacement of the human and equine polyclonal immunoglobulin is advocated and the use of rabies-specific monoclonal antibodies (mAbs) is advocated. We have recently identified a combination of two human monoclonal antibodies (mAbs), CR57 and CR4098, and performed a head-to-head comparison between the $\mathrm{mAb}$ cocktail CR57/CR4098 and human RIG (HRIG). The mAb cocktail neutralized all viruses from a panel of 26 representative natural rabies virus (RV) isolates. The $\mathrm{mAb}$ cocktail in combination with vaccine protected Syrian hamsters from lethal rabies when administered 24 hours after exposure comparable to HRIG. These results demonstrate that the human mAb cocktail CR57/CR4098 is a safe and efficacious alternative for RIG in rabies postexposure prophylaxis (PEP). The antibodies are currently developed using the PER.C6 manufacturing platform and clinical evaluation of the $\mathrm{mAb}$ cocktail will be initiated in the last quarter of 2006.

\section{[15.30-15.50]}

Recombinant human polyclonal antibodies against viruses: A novel therapeutic concept John Haurum

Symphogen A/S, Lyngby, Denmark

Abstract not received.

[15.50-16.10]

Neutralizing antibodies against Venezuelan equine encephalitis virus

Toshiaki Maruyama ${ }^{1}$, Ann R. Hunt ${ }^{2}$, John T. Roehrig $^{2}$, Christopher A. Hinkel, Katherine S. Bowdish ${ }^{1}$, and Shana Frederickson ${ }^{1}$

${ }^{I}$ Alexion Antibody Technologies, Inc., San Diego, CA, USA

${ }^{2}$ Arbovirus Diseases Branch, Division of VectorBorne Infectious Diseases, National Center for Infectious Diseases, Centers for Disease Control and Prevention, Public Health Services, U.S. Department of Health and Human Services, Fort Collins, CO, USA

Venezuelan equine encephalitis virus (VEEV) is a mosquito-borne alphavirus (family Togaviridae) 
maintained in nature by a cycle between insect vector and small rodent hosts. Human infection usually consists of fever, chills, malaise, and severe headache with approximately $1-4 \%$ of cases progressing to severe encephalitis. VEEV has been classified as a "Category B" critical biological agent by the Centers for Disease Control and Prevention (CDC) due to its low human infective dose, easy production, and ability to infect via aerosol. Neutralizing antibodies, either raised by vaccination in animals or passively administered to animal hosts, have been shown to provide protection in animals against VEEV. We have developed a humanized monoclonal antibody (MAb), Hy4 IgG, from a VEEV-neutralizing, protective murine $\mathrm{MAb}$ previously isolated by the CDC. Hy4 IgG was able to passively protect mice either before or after intraperitoneal (i.p.) challenge with VEEV. We also isolated a panel of human Fabs using phage display combinatorial antibody library technology (CoALT) from immunized military personnel. One Fab, F5, may bind to an epitope that is distinct from that recognized by Hy4 IgG. As a fully human Fab, F5 showed good neutralizing ability by plaque reduction neutralizing test (PRNT) and following conversion to whole $\mathrm{IgG}, \mathrm{F} 5$ maintained its neutralizing ability $(70 \%$ neutralization at $10 \mathrm{ng} / \mathrm{ml})$. The human anti-VEEV antibody repertoire will be assessed from the panel of Fabs isolated from the human antibody libraries, in partnership with the CDC as part of the Rocky Mountain Regional Center of Excellence. Additionally, some of these antibodies are strong candidates for use as immunotherapeutics in the treatment of individuals at risk of exposure to VEEV.
[16.10-16.30]

Human-derived, plant-produced monoclonal antibody for the treatment of anthrax

Anna K. Hull ${ }^{1}$, Carolyn J. Criscuolo1, Vadim Mett ${ }^{1}$, Herman Groen2, Wilma Steeman ${ }^{2}$, Hans Westra ${ }^{2}$, Gail Chapman ${ }^{3}$, Bart Legutki ${ }^{3}$, Les Baillie ${ }^{3,4}$ and Vidadi Yusibov ${ }^{1}$

${ }^{I}$ Fraunhofer USA Center for Molecular Biotechnology, 9 Innovation Way Suite 200, Newark, DE 19711, USA

${ }^{2}$ IQ Corporation, Rozenburglaan 13a, 9727 DL Groningen, The Netherlands

${ }^{3}$ Biological Defense Research Directorate, Naval Medical Research Center, 12300 Washington Avenue, Rockville, MD 20852, USA

${ }^{4}$ Medical Biotechnology Center, University of Maryland Biotechnology Institute, 725 West

Lombard Street, Baltimore, MD 21201, USA

The unpredictable nature of bio-terrorism compels us to develop medical countermeasures that will enable authorities to treat individuals exposed to agents such as anthrax. Although, vaccines are proving to be one of the best countermeasures against infectious agents, their efficacy may be limited for immediate post-exposure treatment due to the time required for mounting protective immune responses. The best countermeasure for immediate post-exposure treatment of individuals is the use of monoclonal antibodies that will facilitate direct elimination and spread of threat agent. Combination of both active vaccination and treatment with therapeutic antibodies could be most effective in the case of bio-terrorism attack as well as natural outbreaks. We report the feasibility of producing a protective, human-derived, monoclonal antibody directed against the protective antigen of Bacillus anthracis in plants. This was achieved by transient expression using agroinfiltration of Nicotiana benthamiana plants. The resulting antibody was able to neutralize toxin activity in vitro and protect mice against spore challenge. Moreover, the half-life of plant-made antibody was comparable to that observed for its hybridoma-produced counterpart.

In summary, the results of this study demonstrate that fully active human monoclonal antibodies with specificity to PA can be successfully produced in plants and can be employed for prophylactic use. The study also confirms that the plant-based approach is a viable alternative for manufacturing large quantities of biologically active molecules. 